\title{
Development of multidrug resistance during treatment of isoniazid-resistant tuberculosis
}

\section{To the Editors:}

Several treatment regimens have been recommended for the treatment of isoniazid-resistant tuberculosis (TB). However, an optimal regimen and duration for this treatment remains a matter of some controversy. Here, we would like to share our experience in a case of pulmonary TB with acquired multidrug resistance, during a 12-month treatment of isoniazid-resistant TB with rifampin and ethambutol, with pyrazinamide administered during the initial 2 months.

A 55-yr-old male visited the outpatient chest clinic for the evaluation of a chronic cough. The patient had diabetes mellitus, which had been controlled with oral hypoglycaemic agents. The patient had no history of TB. His chest radiography revealed cavitary consolidation in the left upper lobe. Several sputum samples revealed a host of acid-fast bacilli. Daily antiTB therapy was initiated with isoniazid, rifampin, ethambutol and pyrazinamide. After 2 months of this treatment, the regimen was changed to isoniazid, rifampin and ethambutol, at which time cultured isolates of Mycobacterium tuberculosis were processed for drug susceptibility testing. After 3 months of therapy, the results of drug susceptibility tests indicated high-grade resistance to isoniazid. Isoniazid was discontinued and rifampin and ethambutol were continuously administered on a daily basis. Monthly monitored sputum cultures for acidfast bacilli converted to negative after 2 months of treatment. The total treatment duration was initially scheduled for a full 12 months. After 10 months of therapy, however, an additional sputum culture revealed the growth of 20 colonies of $M$. tuberculosis. A drug-susceptibility test revealed the development of resistance to both isoniazid and rifampin, as well as susceptibility to other drugs. After this, a sputum smear for acid-fast bacilli was, once again, positive.

Previous studies have suggested that standard 6-month, fourdrug regimens may be effective in the treatment of isoniazidresistant TB [1]. In recent years, however, many published guidelines for the treatment of TB have stated that it would be more prudent either to administer pyrazinamide continuously throughout the 6 months, or to prolong the duration of treatment [2-4].

If drug-susceptibility test results are available before the end of the 2 month initial phase of treatment, isoniazid should be discontinued and pyrazinamide should be continued for the entire 6-month duration of therapy (6REZ) [2, 3]. If isoniazid resistance is documented during the 9-month regimen without pyrazinamide, or in the 6-month regimen during the continuation phase of treatment, treatment with rifampin and ethambutol should be continued for a minimum of 12 months (12RE or 2REZ/10RE) [2, 4].

The effectiveness of these recommended regimens has not, until now, been well evaluated. One retrospective study has revealed that a 6-month daily regimen involving the administration of isoniazid, rifampin, ethambutol and pyrazinamide (6HREZ) proved highly effective [5]. However, the effectiveness of the 12-month regimen of rifampin and ethambutol, with or without pyrazinamide during the initial 2 months, has not been evaluated until now.

In patients with isoniazid-resistant tuberculosis, who also have manifested extensive bilateral disease or cavitation on chest radiographs, the development of acquired rifampin resistance could be possible during treatment with rifampin and ethambutol. Our report underlines the seriousness of the concerns regarding the development of multidrug-resistant tuberculosis in patients infected with a Mycobacterium tuberculosis strain with primary isoniazid resistance during treatment with rifampin and ethambutol for 12 months, especially in the cases in which the patient exhibits cavitary pulmonary tuberculosis.

W-J. Koh*, O.J. Kwon*, Y.K. Park ${ }^{\#}$, W.J. Lew ${ }^{\#}$ and G.H. Bai ${ }^{\#}$ *Division of Pulmonary and Critical Care Medicine, Dept of Medicine, Samsung Medical Center, and \#Korean Institute of Tuberculosis, Korean National Tuberculosis Association, Seoul, South Korea.

\section{REFERENCES}

1 Mitchison DA, Nunn AJ. Influence of initial drug resistance on the response to short-course chemotherapy of pulmonary tuberculosis. Am Rev Respir Dis 1986; 133: 423-430.

2 Bass JB Jr, Farer LS, Hopewell PC, et al. American Thoracic Society/Centers for Disease Control and Prevention: treatment and tuberculosis infection in adults and children. Am J Respir Crit Care Med 1994; 149: 1359-1374.

3 Blumberg HM, Burman WJ, Chaisson RE, et al. American Thoracic Society/Centers for Disease Control and Prevention/Infectious Diseases Society of America: treatment of tuberculosis. Am J Respir Crit Care Med 2003; 167: 603-662.

4 Chemotherapy and management of tuberculosis in the United Kingdom: recommendations 1998. Joint Tuberculosis Committee of the British Thoracic Society. Thorax 1998; 53: 536-548.

5 Nolan CM, Goldberg SV. Treatment of isoniazid-resistant tuberculosis with isoniazid, rifampin, ethambutol, and pyrazinamide for 6 months. Int J Tuberc Lung Dis 2002; 6: 952-958. 


\section{Should we use spirometry in the early detection of COPD?}

\section{To the Editors:}

ENRIGHT et al. [1] in the European Respiratory Monograph, promote office spirometry as the way forward in the routine assessment of asthma and chronic obstructive pulmonary disease (COPD), and in the early detection of COPD. They define office spirometry as 'spirometry performed in the primary care setting'. There is an unwelcome ambiguity in their paper when it comes to both of these subjects, and the evidence they use to support their arguments is far from decisive. In the case of the early detection of COPD, the evidence seems to oppose their position.

My first concern is about their use of the term office spirometry, which seems to imply spirometry carried out by the consulting clinician. They say that spirometry with electronic spirometers is now faster than it was with traditional bellows spirometers. This latter suggestion is untrue, even using the $6 \mathrm{~s}$ manoeuvre, since the spirometry manoeuvre is independent of the type of spirometer used, the learning curve for the patient is the same and the instruction given by the operator is also identical. They believe that the main problem with office spirometry is in the quality of the instruction and supervision of the test by the clinician. I agree and think that this must be one of the main objections to spirometry being conducted by clinicians during routine consultations. They quote a primary care Dutch study in which the quality of the spirometry was unacceptably variable [2]. Furthermore, they recommend certification for nurses and technologists carrying out spirometry in primary care. This hardly encourages the routine office use of spirometry by clinicians in their consultations.

The main argument put forward by ENRIGHT et al. [1] is for the use of spirometry in the early detection of COPD. They say that spirometry fulfils all the standard criteria for application of a medical test for screening. A fundamental criterion for any screening programme is the availability of a useful intervention for the patient who screens positive [3]. The main reason to detect COPD in its early stages is to intervene with smoking cessation. ENRIGHT et al. [1] quote three papers in support of the role of early diagnosis of COPD in smoking cessation. None of these actually support their assertion.

The first by RisSER et al. [4] is a trial of a complex intervention comparing education and a motivational intervention with education alone, in which spirometry was a just component of the motivational intervention. In the second paper SEGNAN et al. [5] actually conclude, "In no treatment group was the outcome significantly different from that for one-time counselling at the $(\mathrm{p}<0.05)$ level." In the third paper, GORECKA et al. [6] demonstrated that the diagnosis of airflow limitation had no effect in improving smoking cessation overall, and only in a subanalysis could they show that it leads to an improvement in smoking cessation in those who have moderate or severe airflow limitation. The Global Initiative for Chronic Obstructive Lung Disease (GOLD) guidelines acknowledge the uncertainty surrounding the benefits of community screening of COPD [7]. While there is no argument that smokers should be sought and helped to quit smoking, there is no evidence that early diagnosis of COPD improves smoking cessation.

The promotion of early diagnosis of COPD has been gathering momentum despite the lack of evidence to justify it. Many papers are appearing which report the efforts of clinicians to diagnose COPD early. In the Differential Diagnosis between Asthma and COPD study, BUFFELS et al. [8] report that spirometry-based screening for COPD in primary care doubles the rate of diagnosis of COPD.

The impact on patients and services of a policy to diagnose chronic obstructive pulmonary disease early, which doubles the number of cases in the system, is likely to be expensive and will dilute the resources available for the management of symptomatic chronic obstructive pulmonary disease. It should not even be considered until there is at least some evidence to support it.

\section{P. White}

Dept of General Practice and Primary Care, Guy's, King's and St Thomas' School of Medicine, King's College, London, UK.

\section{REFERENCES}

1 Enright PL, Studnicka M, Zielinski J. Spirometry to detect and manage chronic obstructive pulmonary disease and asthma in the primary care setting. In: Wouters EF, Gosselink R, Stam H, eds. Lung Function Testing. Eur Respir Mon 2005; 31: 1-14.

2 Schermer TR, Jacobs JE, Chavannes NH, et al. Validity of spirometric testing in a general practice population of patients with chronic obstructive pulmonary disease (COPD). Thorax 2003; 58: 861-866.

3 Wilson JM. Principles of screening for disease. Proc $R$ Soc Med 1971; 64: 1255-1256.

4 Risser NL, Belcher DW. Adding spirometry, carbon monoxide, and pulmonary symptom results to smoking cessation counseling: a randomized trial. J Gen Intern Med 1990; 5: 16-22.

5 Segnan N, Ponti A, Battista RN, et al. A randomized trial of smoking cessation interventions in general practice in Italy. Cancer Causes Control 1991; 2: 239-246.

6 Gorecka D, Bednarek M, Nowinski A, Puscinska E, GoljanGeremek A, Zielinski J. Diagnosis of airflow limitation combined with smoking cessation advice increases stopsmoking rate. Chest 2003; 123: 1916-1923. 
7 Pauwels RA, Buist AS, Ma P, Jenkins CR, Hurd SS. Global strategy for the diagnosis, management, and prevention of chronic obstructive pulmonary disease: National Heart, Lung, and Blood Institute and World Health Organization Global Initiative for Chronic Obstructive Lung Disease (GOLD): executive summary. Respir Care 2001; 46: 798-82 (2004 update at www.goldcopd.com).

8 Buffels J, Degryse J, Heyrman J, Decramer M. Office spirometry significantly improves early detection of COPD in general practice: the DIDASCO Study. Chest 2004; 125: 1394-1399.

DOI: $10.1183 / 09031936.05 .00061705$

\section{From the authors:}

Pulmonary specialists welcome increased communication and collaborative work with general practitioners in an attempt to find the most effective evidence-based methods to decrease the substantial morbidity and mortality of asthma and chronic obstructive disorder (COPD). In this issue of the European Respiratory Journal, P. White has highlighted one of the gaps in the evidence base for recommending increased utilisation of spirometry by primary care practitioners. The evidence is indeed weak, in that adding spirometry testing to methods already demonstrated to improve smoking cessation rates (such as counselling, nicotine replacement therapy and bupropion) will further improve the success rates. The published studies were either inadequately designed or had inadequate statistical power to answer this important question. However, several medications to halt the progression of COPD will probably become available in the next few years [1], so we should prepare for them by working to make office spirometry more effective in the primary care setting.

I admit that there are few studies which decisively prove that the addition of spirometry, to the history and physical examination of patients with respiratory symptoms, improves the ability of general practitioners to substantially improve patient-centred outcomes [2]. However, 20 yrs ago, the same could have been said of blood pressure measurements, or blood glucose and haemoglobin-A1c measurements for obese and diabetic patients, respectively. I characterise the tone of our chapter in the European Respiratory Monograph [3] as suggesting "cautious optimism." A major goal of our recommendations is to minimise spirometry misclassification rates, which leads to the many caveats in our recommendations.

When using forced expiratory volume in six seconds reference equations, the average spirometry test session (with a range of 3-8 manoeuvres) is indeed shorter, because the end-of-test criterion for an acceptable manoeuvre is much easier to meet (for children and patients with airway obstruction). Therefore, fewer manoeuvres are needed to meet the goal of three acceptable (including two repeatable) manoeuvres.

In our opinion, whoever coaches the patient to perform spirometry tests needs training and performance-based certification. In the UK, this person is usually the general practitioner (a physician), but in the USA, nurses or technologists often perform the testing. Sometimes a nurse practitioner, physician's assistant, or chronic disease manager uses the results to diagnose or manage the patient.

In Tucson, Arizona, USA, I have seen bumper stickers which say "If I had known that I'd live this long, I would have taken better care of myself." You have probably seen hundreds of patients dying from end-stage COPD, first diagnosed when their forced expiratory volume in one second was $<1 \mathrm{~L}[4]$. How many of them have wondered, "Since my lung disease was apparently slowly progressing for decades before I was finally diagnosed, why didn't anyone tell me about it many years ago? I would have tried much harder to quit smoking." I believe that our time and our limited smoking cessation resources should be preferentially targeted towards patients with the highest risks for smoking-related disease.

In conclusion, epidemiological studies have decisively demonstrated that airway obstruction is the second or third most important risk factor for morbidity and mortality in smoking adults.

\section{P. Enright}

College of Public Health, The University of Arizona, Tucson, AZ, USA.

\section{REFERENCES}

1 Barnes PJ, Stockley RA. COPD: current therapeutic interventions and future approaches. Eur Respir J 2005; 25: 1084-1106.

2 Chavannes N, Schermer T, Akkermans R, et al. Impact of spirometry on GP's diagnostic differentiation and decision-making. Respir Med 2004; 98: 1124-1130.

3 Enright PL, Studnicka M, Zielinski J. Spirometry to detect and manage chronic obstructive pulmonary disease and asthma in the primary care setting. In: Gosselink R, Stam H, eds. Lung Function Testing. Eur Respir Mono 2005; 31: 1-14.

4 Zoia MC, Corsico AG, Beccaria M, et al. Exacerbations as a starting point of pro-active COPD management. Respir Med 2005. 